\title{
Innovations
}

\section{Factors associated with spirit possession: A cross cultural systematic literature review}

\author{
Medical Humanities in the Middle East Conference \\ November 17-18, 2018 \\ Doha, Qatar
}

\section{Authors}

Abdalla A. B. Khairi"*; Aisha A. Abdelkareem²; Bushra Elhusein'

\section{Background}

There are no cross-cultural systematic literature reviews on factors associated with spirit possession. This cross-cultural systematic review included ten primary studies from different countries. The aim is to assess their methodological qualities and summarize the association between spirit possession and certain demographic (gender, age, marital status and educational level) and clinical (hysteria/hysterical traits and anxiety/neuroticism) factors.

\section{Methodology}

An electronic search in relevant databases using suitable search terms was carried out in order to

'Hamad Medical Corporation, Mental Health Hospital, Doha, Qatar

${ }^{2}$ Hamad Medical Corporation, Oncology Hospital, Doha, Qatar „Email: akhairi1@hamad.qa identify studies pertinent to the aims of the review. A large number of studies (710) were identified. Applying inclusion and exclusion criteria, this was reduced to only 10 eligible studies. Studies were included if were in English, address the issue under study, and provide quantitative data on the subject. We excluded essays, case reports, editorials, letters, comments, etc.

\section{Results}

Out of the ten primary studies included in the review, only four $(40 \%)$ were controlled. The remaining six (60\%) were uncontrolled. For this reason, the latter studies were not considered as sources of reliable evidence in the review. According to the controlled studies, the two clinical factors (hysteria/hysterical traits and anxiety/ neuroticism) and one of the demographic factors (educational level) were found to be significantly associated with spirit possession.

\section{Cite this article as:}

Khairi AAB, Abdelkareem AA, Elhusein B. Factors associated with spirit possession: $A$ cross cultural systematic literature review. In: Weber AS, Verjee MA, editors. Proceedings of the 1st International Conference on Medical Humanities in the Middle East [Internet]; 2018 Nov 17-18: Doha, Qatar: Innovations in Global Health Professions Education; 2019 March. p. 47-48. (Innovations in Global Health Professions Education; vol. 2019, spec. no.: 1). https://dx.doi.org/10.20421/ighpe2019.01.16

This is an open access article distributed under the terms of the Creative Commons Attribution license CC BY 4.0, which permits unrestricted use, distribution and reproduction in any medium, provided the original work is properly cited. 


\section{Conclusion}

Firstly, most of the studies in this field lack the methodological rigor necessary to qualify them as reliable bases of evidence. Accordingly, studies are needed that can overcome these methodological difficulties. Secondly, some widely accepted beliefs such as the association of possession with the female gender might be driven by complex socio-cultural factors related to the disadvantaged position of women in some societies. To overcome this, we need to delineate and clearly define these factors by means of appropriate qualitative studies, before we can test the association in question by quantitative studies. Thirdly, it might be reasonable to view the association of low education with possession as a possible indicator of wider socioeconomic disadvantage, and design studies to test this hypothesis. Finally, as for the association of clinical factors (hysteria/hysterical traits and anxiety/neuroticism) with possession, there is need for more precision in future research as distinction between trait and state levels of these factors may add to our knowledge of possession phenomena. Although controlled, the studies that were reviewed are still flawed in many ways (see methodological assessment of controlled studies). These shortcomings were in mind when the phrase "factors associated with...." as opposed to "risk factors of ....." or "determinants of ....." which imply causality, was used in the title of this review. Approached with these limitations in mind, these conclusions would form rich sources for hypothesis construction, and direct and promote future research in this vital field. The orientating tenet would be to, eventually, establish definite causal relationships between possession and various correlates in order to reach a better understanding, classification, diagnosis and treatment.

Conflicts of interest: None.

Funding sources: None.

\section{About the author}

Dr. Abdalla A. B. Khairi is a Consultant Psychiatrist in the Mental Health Hospital, Hamad Medical Corporation, Doha, Qatar. His research interest lies in the field of cultural psychiatry, a career path he followed towards becoming a community activist and literary writer; he has published books and delivered public awareness talks in the field of mental health and other related fields. 